\title{
Long term treatment of recurring pathological fractures due to Mccune Albright Syndrome: Case report and literature review
}

\author{
Yoshvin Sunnassee, Yuhui Shen, Rong Wan, Jianqiang Xu, Weibin Zhang* \\ Department of Orthopedics, Shanghai Ruijin Hospital, Shanghai Jiao Tong University School of Medicine, Shanghai Institute of \\ Traumatology and Orthopedics, Shanghai, China; *Corresponding Author: weibin@medmail.com.cn
}

Received 17 June 2013; revised 15 July 2013; accepted 10 August 2013

Copyright (C) 2013 Yoshvin Sunnassee et al. This is an open access article distributed under the Creative Commons Attribution License, which permits unrestricted use, distribution, and reproduction in any medium, provided the original work is properly cited. In accordance of the Creative Commons Attribution License all Copyrights (C) 2013 are reserved for SCIRP and the owner of the intellectual property Yoshvin Sunnassee et al. All Copyright (C) 2013 are guarded by law and by SCIRP as a guardian.

\begin{abstract}
McCune Albright syndrome is a rare genetic disorder which is characterized by café au lait skin pigmentation, precocious puberty and polyostotic fibrous dysplasia. Treating recurring pathological fractures due to Albright syndrome is a very challenging endeavor, and more so when it is accompanied by poor bone quality and deformity. We hereby present the case of a 23-yearold male patient who is treated several times for recurrent pathological fractures of the femur at our center. We analyze the difficulties associated with treating a patient with poor bone quality over several years, discuss our treatment options, review the literature for similar cases and look at what we could have done differently. We weigh in on the difficulties in treating a severely deformed shepherd's crook, the ways of achieving proper internal fixation and the dangers of using plating instead of an IM nail as suggested in the literature. Our main goal in reporting this case is to bring forth the unusual challenges encountered when treating patients with Albright syndrome and discussing the options of the orthopedic surgeons when treating these types of patients.
\end{abstract}

Keywords: McCune Albright Syndrome; Surgical Treatment; Polyostotic Fibrous Dysplasia

\section{INTRODUCTION}

Mccune Albright syndrome (MAS) is a rare genetic disorder which is characterized by café au lait skin pig- mentation, precocious puberty and polyostotic fibrous dysplasia (PFD) [1,2]. Patients with PFD have poor bone quality and are liable to pathological fractures. We hereby report the case of a male patient who is treated for 7 years in our center for recurring pathological fractures of the femur due to MAS. When the patient was first seen, he presented with a shepherd's crook deformity of the proximal femur. The condition was associated with unequal lower limbs' length, inability to ambulate and pain on motion. He had already undergone several surgeries for recurring femoral neck fractures at another centre. We discuss the challenges of treating this patient, review the literature on the subject and anticipate the challenges that future pathological fractures might pose.

\section{CASE REPORT}

The patient suffered a fracture of the femur while swimming at the age of 10 in 1997 . The fracture did not occur as a result of an impact or a fall. He was treated surgically two times in the pediatrics orthopedics department of another hospital. The first time, bone grafts and a flexible intramedullary nail were used. The fracture did not unite and a subsequent surgery was carried out in 1999 to remedy to the non-united fracture. The exact information of both surgeries and of the post-surgical protocol was not available. The patient still had a painful right lower limb and was dependent on crutches to be able to ambulate. Following the surgery, the patient witnessed weakness in his right lower limb, with pain on ambulation. In 2005, at 16 years of age the patient was first seen at our outpatient department. He presented with a shepherd's crook deformity of the proximal femur, with a fracture in the subtrochanteric region. Physical examination revealed unequaled lower limbs length, difficulty 
ambulating, pain in the right thigh, deformity of the right thigh and dispersed café au lait spots on the abdomen. Roentgenographic examination showed that the patient had several fibrous lesions of the bone in the right proximal femur (Figure 1), craniofacial bone, ribs and bilateral tibia and fibula. The initial diagnosis was Mccune Albright's syndrome with PFD and Type 1 diabetes. He was placed on a course of oral biphosphonates and monitored on a monthly basis. After two years of treatment the patient's dysplastic lesions did not resolve significantly and he was admitted for surgery. Pre-operative tests showed that the patient's blood sugar oscillated between $13 \mathrm{mmol} / \mathrm{L}$ and $18 \mathrm{mmol} / \mathrm{L}$; so we opted for the patient to be discharged. After a course of treatment in our endocrinology department, the daily dose of insulin was re-adjusted. He was re-admitted to our center in 2009 for surgical treatment.

The treatment protocol was limited by several factors which restricted our options:

1. The patient had an unusually small and dysplastic right actebula, when compared to the left side, in which the femoral head could not fit completely.

2. MRI scans showed that a large part of the femoral head was also affected by fibrous dysplasia. This raised the question of improper screw purchase in dysplastic bone. We did not consider the femoral head to be necrotic because the fracture was largely subtrochanteric and would not have affected the blood supply to the femoral head.

3. The bone strip at the point of the fracture, along the shepherd's crook deformity, was very thin which rendered the use of an intramedullary nail very difficult.

4. The diameter of the femur and of the medullary canal was unusually small which would have rendered reaming and the implantation of an intramedullary nail very difficult, with the smallest IM nail available to us being $9 \mathrm{~mm}$ in diameter. A CT examination showed the

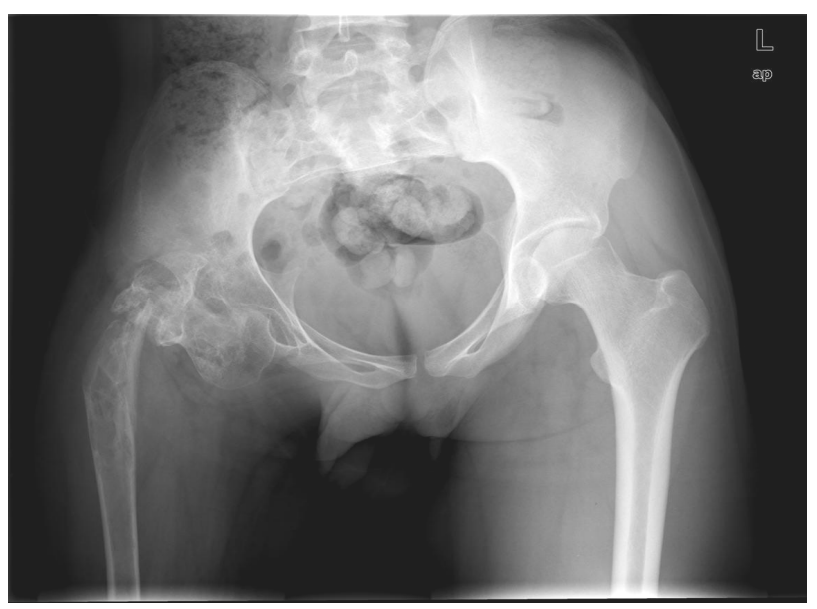

Figure 1. Shows an anterior-posterior view of the pelvis. The right proximal femur has fibrous dysplasia lesions. medullary canal to be also affected by fibrous lesions and was less than $5 \mathrm{~mm}$ in diameter at the point of the lesion and less than $7 \mathrm{~mm}$ diameter distally.

5. Due to the fact that the patient exhibited fibrous bony lesions in the tibia and fibula, we were reluctant to make use of a fibular strut for the corrective osteotomy, as has been suggested by other authors.

6. The patient's severe diabetes could negatively affect bone healing, prompting us to use plating and screws which ensured absolute fixation.

After re-admission, blood sugar level was lower than $14 \mathrm{mmol} / \mathrm{L}$ and a corrective osteotomy with internal fixation and autologous bone grafting was carried out. The intertrochanteric fracture was treated with a DCS plate (Smith \& Nephew, Memphis, Tennessee, United States)c, through a Hardinge approach to the proximal femur. Autologous bone grafts was obtained from the femoral condyles and was used to make up for the considerable bone deficiency at the site of fracture. The patient was given prophylactic antibiotics as post-surgical treatment and was advised to refrain from ambulation. Following the surgery, he was followed at our outpatient department, continued using oral biphosphonates and was able to achieve complete weight-bearing 3 months after the surgery. Subsequent X-rays showed that the fracture united completely after one year and the patient was able to ambulate normally. However, he still had weakness in the affected limb and was only able to walk for a distance of around 50 meters, without resting. The manual muscle test was a grade 4 . Continuous use of biphosphonates did not decrease pain levels.

In November 2011, the patient suffered a fall and he was unable to ambulate. In January 2012, he was once again admitted to our center after roentgenographic scans showed a fatigue fracture of the femur at the distal part of the DCS plate with bending deformity of the femur (Figure 2). The patient was once more treated with an open reduction and internal fixation of the femur. We still faced the same obstacles as during the first surgery but with the added difficulty of having a structurally compromised femur, severe bone deficiency along the fibrous lesions and a femoral head which was both dysplastic and bone deficient. We opted to use a longer plate (DCS, Synthes) and placed the proximal nails in the lower part of the femoral head to allow for better purchase of the nails in normal bone and made up for bony deficiency through bone grafting (Figure 3). The patient was discharged with advice to refrain from ambulation and to undergo rehabilitation under the guidance of the main surgeon. Following the surgery, the patient restarted ambulation after one month and slowly regained the ability to ambulate without walking aids.

The patient was admitted again in April 2012 after he suffered a fracture after another fall. The fracture was 


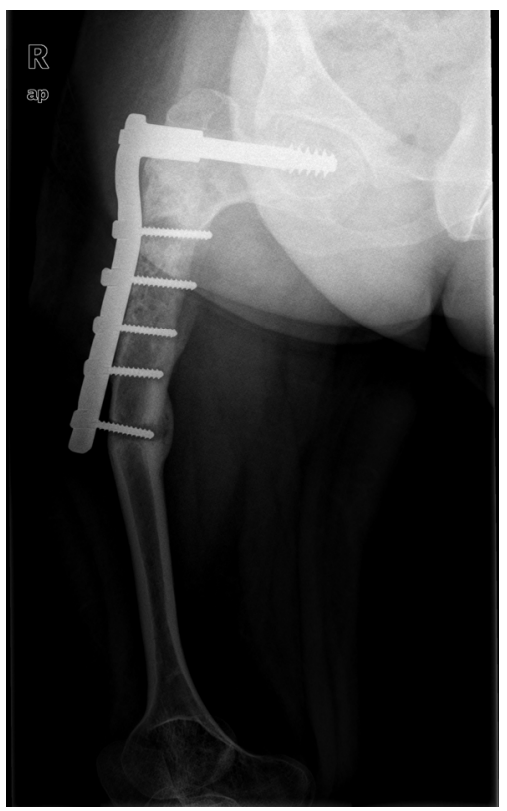

Figure 2. Shows the anterior-posterior view of the femur with bending deformity of the femur at the distal part of the plate.

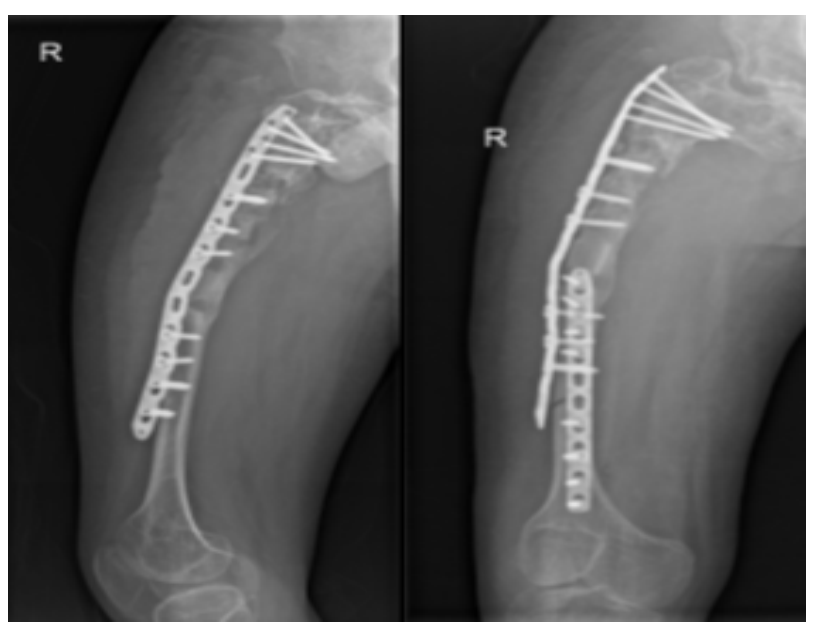

Figure 3. Shows two anterior posterior views of the femur. The left X-ray shows the femur after use of a DCS plate with the proximal nails placed in the lower part of the femoral head. The right X-ray shows the femur after placement of a second plate at the distal part of the femur.

located in the distal part of the femur and was considered to be a result of stress shielding due to the distal part of the DCS plate. We fixed the distal fracture with plating and screws. The fracture line involved a screw which had to be removed and could not be fixed again. The LCDCP plate was placed in the antero-medial part of the femur due to the fact that there was already another plate placed laterally (Figure 3).

At the latest check-up, in June 2012, at 3 months after the last surgery, the patient had regained ambulation, had unequal lower limbs length with the right leg being shorter than the left leg by $3 \mathrm{~cm}$. The patient is still witnessing weakness in the affected limb and is unwilling to make use of corrective shoes. MMT was grade 4 . The fracture healed partially as was seen on follow-up postoperative X-rays.

\section{DISCUSSION}

PFD is a benign bony lesion but can however lead to severe deformity which eventually requires surgery. The shepherd's crook presentation develops as a result of the mechanical load bearing acting on the structurally weakened proximal femoral bone, is a well documented phenomenon [3-5]. Intracapsular fracture may occur due to a shearing force against severe valgus deformity [5] coupled with the fact that the proximal femur has poor bone quality. According to Russell and Chandler, who reported on the surgical treatment of PFD, the indications for surgery in fibrous dysplasia are continued pain in the region of a localized bone lesion, fracture through a lesion, or severe deformity [6]. The medical literature offer very few reports of long term treatment of patient with this disease. Studies have noted that bone dysplasia have normal bone healing capacities [7,8]. Surgical options should take into account the quality of the bone affected and the deformity present. Monostotic fibrous dysplasia, once treated surgically, is unlikely to reoccur while polyostotic fibrous dysplasia, whether it is a presentation of MAS or not, is likely to progress with the disease reaching a quasi-static phase once adulthood is reached [7,9].

Several ways of treating PFD of the proximal femur have been previously discussed in the medical literature, with methods such as intralesional resection and bone grafting or corrective osteotomy with internal fixation which can either be plating or an intramedullary nail. The medical literature supports the idea that in cases of fibrous dysplasia of the proximal femur, an intramedullary device should be used with a screw fixed in the femoral head [10]. The idea of fixing the screw in the femoral head is based on the premise that the femoral head is not affected or rarely affected by fibrous dysplasia and as such can provide proper purchase for screws [9,11,12]. The IM nail can be very useful when dealing with cases of proximal femur PFD with a shepherd's crook deformity, where a wedge osteotomy can be performed to restore the natural geometry of the bone $[3,13,14]$. Corrective osteotomy is particularly useful in cases where there is no fracture of the proximal femur. Success of corrective osteotomy resides on the presence of healthy subtrochanteric bone or in cases where bone grafting is used, autologous healthy bone grafts might be preferred. Jung et al. reported on cases where multiple osteotomies were performed to restore the neck shaft angle using an in- 
tramedullary nail with neck cross-pinning [3]. Furthermore, when we reviewed the literature we found that most instances where IM nails were used were cases with comparatively mild shepherd's crook deformity. Use of plating and screws has been met with mixed success. Chen et al. reported on a case of an intracapsular fracture with shepherd's crook deformity which was treated with corrective osteotomy and internal fixation with a dynamic hip screw. The patient in the report healed completely and did not suffer subsequent fractures [4]. However, other reports have either underlined the risk of bending deformity of the femur or fatigue fracture [3]. According Guile et al., metal plate removal will normally lead to refracture.

The success of plating depends on proper purchase of the screws in healthy bone, which in this case will depend on the state of the femoral head bone and femoral stem bone. Proper purchase guarantees stability of the fixation. It has been noted that restoring the mechanical axis reduces the deforming forced on the bone, with medial displacement varus osteotomy being a possible solution [12]. Decreased neck fracture in PFD patient is a concern and is associated with decreased functional mobility for sports [15]. However, these considerations are at times a luxury in patients with PFD. Restoring ambulation ability remains the goal in most severe cases of polyostotic fibrous dysplasia. Surgical treatments that aim at restoring the proper neck-shaft angle, especially in patients with lower extremity deformity [10] or even in patients with unequal lower limbs length is questionable, because the mechanical axis is not properly restored. An important part of treating PFD induced fracture of the proximal femur is dealing with a structurally compromised stability [7]. Therefore, our choice of surgical protocol mainly focused on allowing the patient to retain ambulation capacity while assuring that the fracture would unite, while having to contour the several restrictions posed by this patient's case.

Intralesional resection and bone grafting was popularized by Enneking [16]. He obtained positive results with this method. However, subsequent studies by other authors revealed that the bone grafts subsequently resolved leaving place to dysplastic lesions. Treatment of PFD with bone grafting, independently of the type of bone strut used or the age of the patient, is unlikely to offer positive results in PFD [7,17]. After the initial surgical treatment in this case, following fractures were all due to trauma. However, even if we were left with no other choices, the use of plating and screws is normally not advised in the treatment of PFD of the proximal femur. As seen in this case, following treatment with plating and screws there was a bending deformity of the proximal femur. It is a controversy in certain cases whether surgery would be the best course of actions, especially in patients with severe bone deformity and deficiency. It has been noted by other authors that in certain cases of PFD, the best approach is to not operate [10], however, we also think that allowing the patient to retain ambulation without walking aids should be a primary goal of PFD surgery of the lower limbs.

The use of biphosphonates in the treatment of PFD has proven its merits with no harmful side-effects, especially when it comes to the ability of bone growth in younger patients [10]. Oral or intravenous biphosphonates both have a positive effect on pain and bone metabolism markers $[18,19]$. However, we noticed that the patient's bone was hard during internal fixation; we cannot confirm whether this change in the quality of bone is directly related to the use of biphosphonates.

Other considerations are important in the peri-operative management of a PFD patient. It is especially important to check bone markers metabolism in the buildup to the surgery. A state of hyperparathyroidism would be an indication against surgical treatment. High levels of parathyroid hormones are likely to signal negative post-surgical results. MAS has been known to develop with endocrinopathies, with patients exhibiting hyperparathyroidism or diabetes with MAS. Even if this patient has type 1-diabetes and his blood sugar level cannot be controlled properly, we chose not to discuss this aspect of the patient's condition as being related to MAS. Even though there has been reports of MAS causing endocrinopathies and a few reports of MAS being related to type 1 diabetes, we could not ascertain that our patient's diabetic condition was a direct result of MAS. It must be noted that the type 1 diabetes represented an obstacle in the peri-operative management of the patient; we think that it should not affect treatment protocol but as is known high blood sugar level can severely affect bone healing.

\subsection{What Would We Do Differently?}

When surgeons face this type recurrent fracture they always need to review their treatment protocol in light of new advances and assess whether they made the right decisions throughout the treatment. These cases of MAS with severe deformity of the proximal femur are always difficult to treat, however, we think that there are a few things that we could consider doing differently.

1. Using a longer plate with fewer screws in the shaft of the femur and placing a cortical screw at the distal end of the plate so as to decrease the stress shielding on the bone would be an operative option that could have benefited the patient. Placing a cortical screw at the distal end has been shown to be beneficial towards decreasing the risk of stress fracture [20]. Using a 3.5 inch pediatric plate instead of a 4.5 inch would also may be have been a 
better fit since the patient's bone was small in size.

2. Placing the femoral neck at an increased valgus angle instead of $90^{\circ}$ varus is also another option but it would have been difficult to achieve due to the very thin strip of bone left along the shepherd's hook. When the patient was operated we placed the femoral neck at a varus angle of $90^{\circ}$, which exposed the non-weight bearing part of the articular cartilage of the femoral head (the more lateral side of the femoral head) to greater loading. Furthermore, the weight from the hip was transferred onto a $90^{\circ}$ varus femoral neck which could have caused instability at the site of fracture, rendering the internal fixation unstable and thus unlikely to heal. The patient should have been advised to refrain from weight bearing until the fracture heals completely. Furthermore, placing the femoral neck at a valgus angle could have helped to slightly lengthen the leg; however this would be limited by the atrophied muscles and by the sciatic nerve.

3. The degree of aggressivity of the treatment in early childhood is an important question when dealing with these types of patients. Funk et al. noted that the progress of the disease subsides, therefore aggressive surgical intervention must be practiced in children to prevent the possibility of difficult procedures in adults [7]. In this specific case, it is difficult to assess whether a more aggressive course of treatment in early childhood would have made any difference in the prognosis.

\subsection{Future Considerations}

Even if at the last follow-up the patient had regained ambulation, we must consider the surgical protocol in cases of subsequent fractures. The quality of bone of the proximal femur is poor, coupled with the fact that plates were used; it is highly likely that the patient will suffer more fatigue fractures at the distal part of the plate. Treating these fractures will be increasingly difficult. He is still at the very beginning of adulthood, and we do not know yet if the fibrous dysplasia will advance or cease to progress. Furthermore, in old age, when osteopenic changes occur, the patient is likely to suffer more fractures and the subsequent treatment protocol will have to take into consideration the impoverished bone quality. This patient's condition will not improve and is likely to become worse in case of more fractures of the proximal femur, unless major reconstructive surgery of the acetabula and of the femur is undertaken, with the use of total length femoral prosthesis. And even then, the difference of $3 \mathrm{~cm}$ between the lower limbs will not be corrected because a drastic increase in the right lower limb length might damage nerves and blood vessels which are already being taxed by the patient's diabetic condition. We hope to be able to continue fixing future fractures with open reduction and internal fixation until the bone quality is too poor or deformity too severe to continue doing so at which point major reconstructive surgery will be required.

\section{CONCLUSION}

The treatment of polyostotic fibrous dysplasia associated with shepherd's crook deformity can represent a considerable challenge to the orthopedic surgeon. Even if common knowledge had indicated the use of IM nails, in our patient's case, the use of an IM nail would have been much more difficult. However, our primary goal of supplying an absolute fixation at the proximal femur to allow for fracture union and allow the patient to reacquire his ambulating capacities in his right leg was attained. In such cases of PFD, the difficulty of treatment lies in the fact that eventual fatigue fractures are difficult to treat and the end-goal is allowing the patient to retain ambulation and remain pain free.

\section{ACKNOWLEDGEMENTS}

We would like to thank Dr Edward P. Southern for his insight and invaluable help in the conception of this work.

\section{REFERENCES}

[1] Albright, F., Butler, A.M., Hampton, A.O. and Smith, P. (1937) Syndrome characterised by osteitis fibrosa disseminata, areas of pigmentation and endocrine dysfunction with precocious puberty in females: Report of five cases. New England Journal of Medicine, 216, 727-746. http://dx.doi.org/10.1056/NEJM193704292161701

[2] McCune, D. (1936) Osteitis fibrosa cystica: The case of a nine-year-old girl who also exhibits precocious puberty, multiple pigmentation of the skin and hyperthyroidism. American Journal of Diseases of Children, 52, 743-744.

[3] Jung, S.T., Chung, J.Y., Seo, H.Y., Bae, B.H. and Lim, K.Y. (2006) Multiple osteotomies and intramedullary nailing with neck cross-pinning for shepherd's crook deformity in polyostotic fibrous dysplasia: 7 femurs with a minimum of 2 years follow-up. Acta Orthopadica, 77, 469-473. http://dx.doi.org/10.1080/17453670610046415

[4] Chen, W.J., Chen, W.M., Chiang, C.C., Huang, C.K., Chen, T.H. and Lo, W.H. (2005) Shepherd's crook deformity of polyostotic fibrous dysplasia treated with corrective osteotomy and dynamic hip screw. Journal of the Chinese Medical Association, 68, 343-346. http://dx.doi.org/10.1016/S1726-4901(09)70173-X

[5] Tsuchiya, H., Tomita, K., Matsumoto, T. and Watanabe, S. (1995) Shepherd's crook deformity with an intracapsular femoral neck fracture in fibrous dysplasia. Clinical Orthopaedics and Related Research, 310, 160-164. http://dx.doi.org/10.1097/00003086-199501000-00025

[6] Russell, L.W. and Chandler, F.A. (1950) Fibrous dysplasia of bone. The Journal of Bone \& Joint Surgery [American], 32, 323-337.

[7] Funk Jr., F.J. and Wells, R.E. (1973) Hip problems in 
fibrous dysplasia. Clinical Orthopaedics, 90, 77-82.

[8] Harris, W.H., Dudley Jr., H.R. and Barry, R.J. (1962) The natural history of fibrous dysplasia: An orthopaedic, pathological, and roentgenographic study. The Journal of Bone \& Joint Surgery [American], 44, 207-233.

[9] Ozaki, T., Sugihara, M., Nakatsuka, Y., Kawai, A. and Inoue, H. (1996) Polyostotic fibrous dysplasia: A long term follow up of 8 patients. International Orthopaedics, 20, 227-232. http://dx.doi.org/10.1007/s002640050069

[10] Leet, A. and Collins, M.T. (2007) Current approach to fibrous dysplasia of bone and Mc-Cune Albright syndrome. Journal of Children's Orthopaedics, 1, 3-17. http://dx.doi.org/10.1007/s11832-007-0006-8

[11] Harris, W.H., Dudley Jr., H.R. and Barry, R.J. (1962) The natural history of fibrous dysplasia: An orthopaedic, pathological, and roentgenographic study. The Journal of Bone \& Joint Surgery [American], 44, 207-233.

[12] Guille, J.T., Kumar, S.J. and MacEwen, G.D. (1998) Fibrous dysplasia of the proximal part of the femur: Longterm results of curettage and bone-grafting and mechanical realignment. The Journal of Bone \& Joint Surgery [American], 80, 648-658.

[13] Ippolito, E., Caterini, R., Farsetti, P. and Potenza, V. (2002) Surgical treatment of fibrous dysplasia of bone in McCune. Journal of Pediatric Endocrinology and Metabolism, 15, 939-944.

[14] O’Sullivan, M. and Zacharin, M. (2002) Intramedullary rodding and bisphosphonate treatment of polyostotic fibrous dysplasia associated with the McCune-Albright syndrome. Journal of Pediatric Orthopaedics, 22, 255-
260. http://dx.doi.org/10.1097/01241398-200203000-00025

[15] Leet, A.I., Wientroub, S., Kushner, H., Brillante, B., Kelly, M.H., Robey, P.G., et al. (2006) The correlation of specific orthopaedic features of polyostotic fibrous dysplasia with functional outcome scores in children. The Journal of Bone \& Joint Surgery [American], 88, 818-823. http://dx.doi.org/10.2106/JBJS.E.00259

[16] Enneking, W.F. and Gearen, P. (1986) Fibrous dysplasia of the femoral neck. Treatment by cortical bone-grafting. The Journal of Bone \& Joint Surgery [American], 68, 1415-1422.

[17] Guille, J.T., Kumar, S.J. and MacEwen, G.D. (1998) Fibrous dysplasia of the proximal femur. Long-term results of curettage and bone-grafting and mechanical realignment. The Journal of Bone \& Joint Surgery [American], 80, 648-658.

[18] Weinstein, R. (1997) Long-term aminobisphosphonate treatment of fibrous dysplasia: Spectacular increase in bone density. Journal of Bone and Mineral Research, 12, 13141315. http://dx.doi.org/10.1359/jbmr.1997.12.8.1314

[19] Lane, J.M., Khan, S.N., O’Connor, W.J., Nydick, M., Hommen, J.P., Schneider, R., et al. (2001) Bis-phosphonate therapy in fibrous dysplasia. Clinical Orthopaedics, 382, 6-12. http://dx.doi.org/10.1097/00003086-200101000-00003

[20] Bottlang, M., Doornink, J., Fitzpatrick, D.C. and Madey, S.M. (2009) Far cortical locking can reduce stiffness of locked plating constructs while retaining construct strength. The Journal of Bone \& Joint Surgery [American], 91, 1985-1994. http://dx.doi.org/10.2106/JBJS.H.01038 